Letter to the Editor

\title{
Validation data is needed to support modelling in Road Ecology
}

Roads and other linear transportation infrastructures are among the most ubiquitous human-made features on the globe, causing several pernicious negative effects on biodiversity including mortality due to collisions and acting as barriers to animal movement and gene flow. While there are several studies describing such negative effects in a large collection of species, very few attempted to quantify the consequences of such impacts on the biodiversity, in particular in population persistence. Recently, BC published an interesting exercise by Pinto et al. (2018) aiming to assess the relative role of habitat fragmentation and additional mortality due to collisions with vehicles on population viability of giant anteater (Myrmecophaga tridactyla) in Brazil. The work is based on the theoretical models developed by Bordade-Água et al. (2011), which aim to provide minimum patch size $\left(\mathrm{P}_{\min }\right)$ and critical road density $\left(D_{\max }\right)$ threshold values for species persistence. This is a value information for assisting on planning ecologically sustainable road networks, particularly in times when a massive expansion of transportation infrastructures is foreseen (Meijer et al., 2018), with potential dramatic consequences for biodiversity.

However, and this is our main point, such a modeling exercise lacks a validation procedure to inform the accuracy and robustness of the transferability of the theoretical models developed by Borda-de-Água et al. (2011) when applied to this specific system. Recently, Ceia-Hasse et al. (2017) presented a modelling study aimed to assess the effects of transportation infrastructures on carnivore population persistence at the global scale. Although also lacking validation, such exercise covering the global scale can inform broad planning directives, namely calling the attention to the importance of roads to the conservation of large mammals. In this case, the specificity of the species and study area makes it very audacious to present such results without a validation component. By reporting threshold values on key indicators such as $\mathrm{P}_{\min }$ and $\mathrm{D}_{\max }$, authors are potentially influencing the conservation guidelines for that Vulnerable species. Yet, without a clear notion on the uncertainty and validity of the model applied to this species, those thresholds might be heavily biased, hence opening the door to wrong management practices, including those targeting the species conservation and those determining consequent human development 'allowed' in respect of those indicators. For example, the authors conclude that "habitat fragmentation has a greater impact on persistence of the giant anteater population in Brazil than the observed mortality due to vehicle collisions". This simple statement can lead road managers to neglect roadkill mitigation measures, such as traffic speed reduction (highly effective and a less expensive solution), and favor building wildlife crossing structures (a more expensive measure). However, expensive measures are often more difficult to implement and replicate, and so one might end with no mitigation targeting roadkill reduction and few measures to reduce the barrier effect.

It is true that the work presents a sensitivity analysis to the key parameters to estimate $P_{\min }$ and $D_{\max }$, namely dispersal and population density, but this is not validation. Moreover, authors used model assumptions highly unrealistic for the giant anteater. In particular, to estimate $\mathrm{P}_{\min }$ they assumed an infinite carrying capacity, an exponential growth in suitable habitats and, perhaps more confusing, that all individuals die when crossing a road. They support this last assumption by stating that the animals move at slow speed, which is arguably; and by the observation of high roadkill rates, which is true but far from implying that the majority of individuals crossing roads are killed. On the other hand, to estimate $D_{\max }$ they assumed an exponential population growth, an infinite dispersal, and an infinite carrying capacity. These assumptions are in line with the theoretical exercise developed by Borda-de-Água et al. (2011), but can hardly be supported in this concrete species, especially infinite dispersal which is a key parameter in the theoretical models. In fact, Borda-de-Água et al. (2011) clearly affirm that although their estimate of $D_{\max }$ provides an easy rule of thumb to assess the viability of a population on the basis of growth rates and road density, it is only a first approximation. Hence, despite the usefulness of the theoretical model, its applicability still requires validation grounded on empirical data.

Overall, the lack of validation in Road Ecology modelling is worth discussing, as it is a serious handicap in this discipline - transversal to other applied ecology branches -, with potential harmful consequences for biodiversity conservation. Research in Road Ecology should strive to develop transferability assessments within the modelling framework to validate the use of theoretical or other simulation models (Wenger and Olden, 2012). Only then we can judge the predictive power and hence the credibility of the models. The models presented by Borda-de-Água et al. (2011) are an excellent example of a theoretical work that still needs a last step of validation to become a powerful tool to analyze the impact of transport infrastructures on biodiversity. Generally, such demonstration involves a comparison of model outputs with data obtained by observation and measurement of the real system, i.e. empirical-based data. In the study under consideration, different options were available, including comparing the roadkill rates in areas classified by Pinto et al. (2018) as 'Critical areas for species persistence' with those in areas of 'Conservation opportunities'. Another option would be to compare population densities - or other proxy such as (camera) trapping success - between those two area types. The ongoing conservation program on the giant anteater (www.giantanteater.org) in Mato Grosso do Sul (Brazil), involving leading experts on this species, can help attesting the robustness of their main outputs in future research.

By including a validation procedure in modelling, Road Ecology can provide more robust and less biased tools that can assist both landscape and road managers, as well as conservation dedicated professionals in the planning and mitigation of transportation infrastructures.

\section{Acknowledgements}

We appreciate the comments and contributions of Luís Borda-de- 
Água to a previous version of this text.

\section{References}

Borda-de-Água, L., Navarro, L., Gavinhos, C., Pereira, H.M., 2011. Spatio-temporal impacts of roads on the persistence of populations: analytic and numerical approaches. Landsc. Ecol. 26, 253-265. https://doi.org/10.1007/s10980-010-9546-2.

Ceia-Hasse, A., Borda-de-Água, L., Grilo, C., Pereira, H.M., 2017. Global exposure of carnivores to roads. Glob. Ecol. Biogeogr. 26, 592-600. https://doi.org/10.1111/geb. 12564.

Meijer, J.R., Huijbregts, M.A.J., Schotten, K.C.G.J., Schipper, A.M., 2018. Global patterns of current and future road infrastructure. Environ. Res. Lett. 13, 064006. https://doi. org/10.1088/1748-9326/aabd42.

Pinto, F.A.S., Bager, A., Clevenger, A.P., Grilo, C., 2018. Giant anteater (Myrmecophaga tridactyla) conservation in Brazil: analysing the relative effects of fragmentation and mortality due to roads. Biol. Conserv. 228, 148-157. https://doi.org/10.1016/j. biocon.2018.10.023.

Wenger, S.J., Olden, J.D., 2012. Assessing transferability of ecological models: an underappreciated aspect of statistical validation. Methods Ecol. Evol. 3, 260-267. https://doi.org/10.1111/j.2041-210X.2011.00170.x.
Fernando Ascensão ${ }^{\mathrm{a}, \mathrm{b}, \mathrm{c}, *}$, Marcello D'Amico ${ }^{\mathrm{d}}$, Rafael Barrientos ${ }^{\mathrm{e}}$ ${ }^{a}$ CIBIO/InBio, Centro de Investigação em Biodiversidade e Recursos Genéticos, Universidade do Porto, Portugal

${ }^{\mathrm{b}}$ Centro de Ecologia Aplicada "Professor Baeta Neves" (CEABN), InBio, Instituto Superior de Agronomia, Universidade de Lisboa, Portugal ${ }^{\mathrm{c}}$ Department of Conservation Biology, Estación Biológica de Doñana (EBDCSIC), Sevilla, Spain

${ }^{\mathrm{d}}$ Department of Environmental Chemistry, Institute of Environmental Assessment and Water Research IDAEA (CSIC), Calle Jordi Girona, 18-26, 08034 Barcelona, Spain

${ }^{\mathrm{e}}$ Universidad de Alcalá, Departamento de Ciencias de la Vida - Área de Ecología, Edificio de Ciencias Campus Universitario, Carretera MadridBarcelona, km 33.6 E-28871, Alcalá de Henares, Madrid, Spain E-mail address: fjascensao@cibio.up.pt (F. Ascensão).

${ }^{*}$ Corresponding author at: CIBIO/InBio, Centro de Investigação em Biodiversidade e Recursos Genéticos, Universidade do Porto, Portugal. 